Geometry \& $\mathcal{T}$ Topology

Volume 1 (1997) 1-7

Published: 7 February 1997

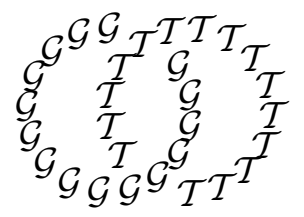

\title{
Groups acting on CAT(0) cube complexes
}

\author{
Graham Niblo \\ LAWRENCE REEVES \\ Faculty of Mathematical Studies \\ University of Southampton \\ Highfield \\ Southampton, SO17 1BJ, UK \\ and \\ Institute of Mathematics \\ Hebrew University of Jerusalem \\ Givat Ram \\ Jerusalem 91904, Israel \\ Email: gan@maths.soton.ac.uk and lawrence@math.huji.ac.il
}

\begin{abstract}
We show that groups satisfying Kazhdan's property $(\mathrm{T})$ have no unbounded actions on finite dimensional $\mathrm{CAT}(0)$ cube complexes, and deduce that there is a locally $\operatorname{CAT}(-1)$ Riemannian manifold which is not homotopy equivalent to any finite dimensional, locally $\mathrm{CAT}(0)$ cube complex.
\end{abstract}

\section{AMS Classification numbers Primary: $20 \mathrm{~F} 32$}

Secondary: 20E42, 20G20

Keywords: Kazhdan's property (T), Tits' buildings, hyperbolic geometry, CAT $(0)$ cube complexes, locally CAT $(-1)$ spaces, $S p(n, 1)$-manifolds

Proposed: Walter Neumann

Seconded: David Gabai, Robion Kirby
Received: 28 October 1996

Accepted: 6 February 1997

Copyright Geometry and Topology 


\section{Introduction}

The $\operatorname{CAT}(\chi)$ inequality gives a measure of the curvature of a geodesic metric space $X$ by comparing the width of the geodesic triangles in $X$ with those of the corresponding triangles in the simply connected Riemannian manifold of constant curvature $\chi$. The theory of $\operatorname{CAT}(\chi)$ metric spaces is described in [2].

A geodesic metric space $X$ is said to be locally $\operatorname{CAT}(\chi)$ if around every point there is a non-empty ball which is geodesically convex and $\operatorname{CAT}(\chi)$. It follows from the definition that $X$ is locally $\operatorname{CAT}(0)$ if every point has a $\operatorname{CAT}(\chi)$ neighbourhood for some $\chi$ less than or equal to 0 , where $\chi$ is allowed to vary with the point.

Examples of locally CAT(0) spaces are furnished by Riemannian manifolds whose sectional curvatures are all bounded above by 0 , by what Davis calls in [4] the "(correctly defined) geometric realization" of a Tits' building, and by compact piecewise Euclidean polyhedral complexes satisfying certain curvature conditions on the link of every cell, as studied by Bridson in his thesis, [1]. If the cells of such a complex are all isometric to Euclidean cubes then the curvature condition can be expressed in terms of the combinatorics of the links of the cells (Gromov's "no bigon" and "no triangle" conditions [5], outlined here in section 3). It is an open problem to decide which CAT( 0$)$ spaces can be given a piecewise Euclidean polyhedral structure which is also CAT( 0$)$.

In this paper we establish the following theorem:

Theorem A For any $n \geq 2$ there is a smooth locally CAT(-1) Riemannian manifold $M$ of dimension $4 n$ which is not homotopy equivalent to any finite dimensional, locally CAT( 0$)$ cube complex.

The manifold $M$ is a quotient of quaternionic hyperbolic space $\mathbb{O} \mathbb{H}^{n}$ (of real dimension $4 n$ ) by a discrete group $G$ of isometries. The isometry group of $\mathbb{O H}^{n}$ is the symplectic group $S p(n, 1)$; in fact we may choose $G$ to be any discrete cocompact lattice in $S p(n, 1)$, and let $M$ be the corresponding quotient of $\mathbb{O H}^{n}$, so $G=\pi_{1}(M)$. Now if $M$ was homotopy equivalent to a compact, locally CAT $(0)$ cube complex $X$, then $G$ would act freely and properly discontinuously on the universal cover $\tilde{X}$ which is a non-compact finite dimensional $\operatorname{CAT}(0)$ cube complex (see [1] for a discussion of how the local curvature properties of $X$ give global curvature constraints on the universal cover). We will see that $G$ admits no such actions; indeed we will show that any cellular action of $G$ on a finite dimensional CAT $(0)$ cube complex must have a global fixed point. This will follow from the fact that $G$ has Kazhdan's property (T) [6], together with the following result:

Geometry and Topology, Volume 1 (1997) 
Theorem B If $G$ is a group satisfying Kazhdan's property $(\mathrm{T})$ and $X$ is a finite dimensional CAT(0) cube complex on which $G$ acts cellularly (and therefore isometrically), then the action has a global fixed point.

We note that since $M$ is a compact locally $\operatorname{CAT}(-1)$ space, $\pi_{1}(M)$ is a $\delta$ hyperbolic group, and so this gives an example of a $\delta$-hyperbolic group which cannot act without fixed points on any finite dimensional CAT(0) cube complex.

Further examples of $T$-groups are furnished by groups acting simply transitively on Tits' buildings modelled on the $\tilde{A}_{2}$ simplex, ie the Euclidean triangle with angles $\pi / 4, \pi / 4$ and $\pi / 2,[6]$. As Davis shows in [4], these buildings are $\mathrm{CAT}(0)$, so their quotients are locally $\mathrm{CAT}(0)$. Their quotients give examples of locally $\mathrm{CAT}(0)$ simplicial complexes which are not homotopy equivalent to any finite dimensional, locally $\mathrm{CAT}(0)$ cube complexes.

The proof of Theorem B is modelled on (or stolen from, depending on your point of view) the proof by Bozejko et al [3] that finitely generated Coxeter groups do not have property $(\mathrm{T})$. It can be shown [7] that finitely generated Coxeter groups act effectively, properly discontinuously and cellularly on finite dimensional CAT(0) cube complexes so Theorem A may be viewed as a generalisation of their result.

In section 2 we will recall the definition of a "conditionally negative kernel" on a group as given in [6] and the characterisation of Kazhdan's property (T) in terms of these kernels. We will then outline the proof of Theorem B. In section 3 we will give the definition of a CAT( 0 ) cube complex and derive the technical result we need to complete the missing steps of the proof.

\section{Kazhdan's property (T)}

In [6] de la Harpe and Valette give several equivalent formulations of Kazhdan's property $(\mathrm{T})$ for locally compact groups. Although it is not the most intuitive one we will use the following criterion to establish our main result:

Definition A conditionally negative kernel on a set $V$ is a function $f: V \times$ $V \longrightarrow \mathbb{R}$ such that for any finite subset $\left\{v_{1}, \ldots, v_{n}\right\} \subset V$ and any real numbers $\left\{\lambda_{1}, \ldots, \lambda_{n}\right\}$ such that $\sum_{i} \lambda_{i}=0$ the following inequality holds:

$$
\sum_{i, j} \lambda_{i} \lambda_{j} f\left(v_{i}, v_{j}\right) \leq 0
$$

Geometry and Topology, Volume 1 (1997) 
A conditionally negative kernel on a group $G$ is a conditionally negative kernel on the set of elements of $G$ such that for any $g, h, k$ in $G, f(g h, g k)=f(h, k)$.

According to [6] a finitely generated group $G$ has Kazhdan's property (T) (or is a $T$-group) if and only if every conditionally negative kernel on $G$ is bounded.

Our goal is to prove the following theorem. The statement is followed by an outline proof and the missing lemma is given in the following section.

Theorem B If $G$ is a group satisfying Kazhdan's property $(\mathrm{T})$ and $X$ is a finite dimensional CAT $(0)$ cube complex on which $G$ acts cellularly (and therefore isometrically), then the action has a global fixed point.

Proof In section 3 we will show that for any CAT(0) cube complex $X$, the simplicial metric $D$ on the 1 -skeleton of $X$ restricts to a conditionally negative kernel on the vertex set $X^{(0)}$. Since $G$ acts cellularly on $X, D$ is invariant under the action, so setting $f(g, h)=D(g v, h v)$ gives a conditionally negative kernel on the group $G$. Since $G$ has Kazhdan's property (T) the conditionally negative kernel must be bounded so the orbit $G v$ is bounded in the metric $D$ and therefore, since $X$ is finite dimensional, in the metric $d$ (see section $3)$. Since $X$ is finite dimensional it is complete [1] and in a complete CAT $(0)$ metric space any isometric action with a bounded orbit has a global fixed point ([1] again), completing the proof of Theorem B.

\section{CAT(0) cube complexes}

A cube complex $X$ is a metric polyhedral complex in which each cell is isometric to the Euclidean cube $[-1 / 2,1 / 2]^{n}$, and the gluing maps are isometries. If there is a bound on the dimension of the cubes then the complex carries a complete geodesic metric, [1].

Definition A cube complex is non-positively curved if for any cube $C$ the following conditions on the link of $C, l k(C)$, are satisfied:

(i) (no bigons) For each pair of vertices in $l k(C)$ there is at most one edge containing them.

(ii) (no triangles) Every edge cycle of length three in $l k(C)$ is contained in a 2 -simplex of $l k(C)$.

The following theorem of Gromov relates the combinatorics and the geometry of the complex.

Geometry and Topology, Volume 1 (1997) 
Lemma (Gromov, [5]) A cube complex $X$ is locally CAT(0) if and only if it is non-positively curved, and it is $\mathrm{CAT}(0)$ if and only if it is non-positively curved and simply connected.

Examples Any graph may be regarded as a 1-dimensional cube complex, and the curvature conditions on the links are trivially satisfied. The graph is CAT $(0)$ if and only if it is a tree. Euclidean space also has the structure of a CAT $(0)$ cube complex with its vertices at the integer points.

A midplane of a cube $[-1 / 2,1 / 2]^{n}$ is its intersection with a codimension 1 coordinate hyperplane. So every $n$-cube contains $n$ midplanes each of which is an $(n-1)$-cube, and any $m$ of which intersect in a $(n-m)$-cube. Given an edge in a non-positively curved cube complex, there is a unique codimension 1 hyperplane in the complex which cuts the edge transversely in its midpoint. This is obtained by developing the coordinate hyperplanes in the cubes containing the edge. In the case of a tree the hyperplane is the midpoint of the edge, and in the case of Euclidean space it is a geometric hyperplane.

In general the hyperplane is analogous to an immersed codimension 1 submanifold in a Riemannian manifold and the immersion is actually a local isometry. An application of the Cartan-Hademard theorem, [5, section 4], then shows that the hyperplane is isometrically embedded, and furthermore any hyperplane in a CAT(0) cube complex separates it into two components referred to as the half spaces associated with the hyperplane. This is a consequence of the fact that the complex is simply connected. The hyperplane gives rise to a 1-cocycle which is necessarily trivial, and hence the hyperplane separates the space.

The set of vertices of the cubing $X$ can be viewed as a discrete metric space, where the metric $D(u, v)$ is given by the length of the shortest edge path between the vertices $u$ and $v$ in the 1 -skeleton of $X$. If $X$ is finite dimensional this metric is quasi-isometric to the $\operatorname{CAT}(0)$ metric (it is at most $\sqrt{n}$ times the CAT( 0$)$ metric, where $n$ is the dimension of the complex; this does not require the complex to be cocompact). Sageev, [8], observed that the shortest path in the 1-skeleton crosses any hyperplane at most once, and since every edge crosses exactly one hyperplane, the distance between two vertices is the number of hyperplanes separating them. Recalling that a hyperplane separates the vertices into two half spaces $U^{+}$and $U^{-}$, it follows that

$$
D(u, v)=\sum_{U} \chi_{U}(u)\left(1-\chi_{U}(v)\right)
$$

where $\chi_{U}$ is the characteristic function of the half space $U$, and $U$ ranges over all the half spaces in $X$. Although there are infinitely many half spaces, the 
fact that only finitely many of them separate any pair of vertices means that this will be a finite sum.

Now suppose that $G$ is a group acting cellularly on $X$. The action restricts to the 1-skeleton and therefore preserves the metric $D$, so we have established parts 2 and 3 of the following lemma:

Technical Lemma For any CAT(0) cube complex $X$ the simplicial metric $D$ on the 1-skeleton of $X$ satisfies the following properties:

1) $D$ is a conditionally negative kernel on the vertex set of $X$.

2) $D$ is invariant under the action of $G$ on the vertex set.

3) For any vertices $u, v \in X, d(u, v) \leq D(u, v) \leq \sqrt{n} d(u, v)$ where $n$ is the dimension of the cube complex.

Proof It remains to establish that the metric $D$ is a conditionally negative kernel. Let $u_{1}, u_{2}, \ldots, u_{n} \in G$ and $\lambda_{i} \in \mathbb{R}$ with $\sum_{i} \lambda_{i}=0$. Then

$$
\sum_{i} \sum_{j} \lambda_{i} \lambda_{j} D\left(u_{i}, u_{j}\right)=\sum_{i} \sum_{j} \lambda_{i} \lambda_{j} \sum_{U} \chi_{U}\left(u_{i}\right)\left(1-\chi_{U}\left(u_{j}\right)\right)
$$

where the third sum can taken over the finitely many half spaces $U$ separating the vertices $\left\{u_{1}, \ldots, u_{n}\right\}$ (the other half spaces all contribute 0 to the sum). This triple sum can now be rearranged to get

$$
\sum_{i} \sum_{j} \lambda_{i} \lambda_{j} \sum_{U} \chi_{U}\left(u_{i}\right)-\sum_{i} \sum_{j} \lambda_{i} \lambda_{j} \sum_{U} \chi_{U}\left(u_{i}\right) \chi_{U}\left(u_{j}\right)
$$

The first term is zero since it can be rearranged to give $\sum_{j} \lambda_{j} \sum_{i} \lambda_{i} \sum_{U} \chi_{U}\left(u_{i}\right)$, and $\sum_{j} \lambda_{j}=0$ by hypothesis. Likewise the second term can be rewritten as

$$
\sum_{U} \sum_{i} \lambda_{i} \chi_{U}\left(u_{i}\right) \sum_{j} \lambda_{j} \chi_{U}\left(u_{j}\right)=\sum_{U}\left(\sum_{i} \lambda_{i} \chi_{U}\left(u_{i}\right)\right)^{2}
$$

which is positive. It follows that the entire sum is negative as required. 


\section{References}

[1] M Bridson, Geodesics and curvature in metric simplicial complexes, from: "Group Theory from a Geometrical Viewpoint", E Ghys et al (eds.), World Scientific (1991) 373-463

[2] M Bridson, A Haefliger, Metric spaces of non-positive curvature, in preparation

[3] M Bozejko, T Janusckiewicz, R T Spatzier, Infinite Coxeter groups do not have Kazhdan's property, J. Operator Theory 19 (1988) 63-37

[4] M Davis, Buildings are CAT(0), from: "Geometric methods in group theory", P H Kropholler, G A Niblo and R Stohr (eds.), LMS Lecture Note Series, Cambridge University Press

[5] M Gromov, Hyperbolic groups, from: "Essays in group theory", S M Gersten (ed.), MSRI Publ. 8, Springer-Verlag (1987) 75-267

[6] $\mathbf{P}$ de la Harpe, A Valette, La propriété (T) de Kazhdan pour les groupes localement compacts, Asterisque 175 (1989), Société Mathématique de France

[7] G A Niblo, L D Reeves, Coxeter groups act on CAT(0) cube complexes, preprint

[8] M Sageev, Ends of group pairs and non-positively curved cube complexes, Proc. London Maths. Soc. (3) 71 (1995) 585-617

Geometry and Topology, Volume 1 (1997) 